\title{
Single-Resistance Controlled Sinusoidal Oscillator Employing Single Universal Voltage Conveyor
}

\author{
Kanhaiya Lal Pushkar \\ Department of Electronics and Communication Engineering, Maharaja Agrasen Institute of Technology, New Delhi, India \\ Email: klpushkar17@gmail.com
}

How to cite this paper: Pushkar, K.L. (2018) Single-Resistance Controlled Sinusoidal Oscillator Employing Single Universal Voltage Conveyor. Circuits and Systems, 9, 1-7.

https://doi.org/10.4236/cs.2018.91001

Received: December 9, 2017

Accepted: January 22, 2018

Published: January 25, 2018

Copyright $\odot 2018$ by author and Scientific Research Publishing Inc. This work is licensed under the Creative Commons Attribution International License (CC BY 4.0).

http://creativecommons.org/licenses/by/4.0/

\begin{abstract}
In this paper, a new single-resistance controlled sinusoidal oscillator (SRCO) using single universal voltage conveyor (UVC) has been presented. The proposed SRCO employs single universal voltage conveyor, three resistors, and two capacitors. The proposed configuration offers the following advantageous features (1) independent control of condition of oscillation and frequency of oscillation (2) low passive sensitivities. The validity of the proposed SRCO has been established by SPICE (version 16.5) simulations using Taiwan Semiconductor Manufacturing Company (TSMC) $0.18 \mu \mathrm{m}$ technology.
\end{abstract}

\section{Keywords}

Universal Voltage Conveyor, SRCO, Sinusoidal Oscillator

\section{Introduction}

Implementation of sinusoidal oscillators and active biquadratic filters has become important modern analog active building blocks that have been introduced in [1], and UVC is one of them which is emerging as a very flexible and versatile building block for analog signal processing and has been used earlier for realizing a number of functions. SRCOs play an important role in control systems, signal processing, communication, and instrumentation and measurement systems [2]-[7]. The applications, advantages, and usefulness of UVC have now been recognized in the realization of filters, in inductance simulation and in the realization of sinusoidal oscillator [8]-[15]. In ref. [16], authors have been presented third-order quadrature oscillator using two second generation current conveyors, a UVC, three capacitors and three resistors. However, to the best of 
the knowledge and belief of the author, none of the SRCOs using single UVC has yet been presented in the literature so far. Therefore, the purpose of this paper is to present a new SRCO using a single UVC, two capacitors and three resistors. The proposed structure offers (1) independent control of both frequency of oscillation and, condition of oscillation (2) low passive sensitivities. The validity of the proposed SRCO has been confirmed by SPICE simulation using 0.18 $\mu \mathrm{m}$ TSMC process parameters.

\section{New Oscillator Configuration}

The symbolic notation and equivalent circuit model of UVC are shown in Figure 1(a) and Figure 1(b) respectively. The UVC is a 6-port active element with one voltage input $x$, two difference current inputs $\left(y^{+}, y^{-}\right)$, two mutually inverse voltage outputs $\left(z^{+}, z^{-}\right)$, and one auxiliary port $w$. The UVC can be described by the following set of equations.

$$
\left(\begin{array}{c}
I_{x} \\
I_{w} \\
V_{y^{+}} \\
V_{y^{-}} \\
V_{z^{+}} \\
V_{z^{-}}
\end{array}\right)=\left(\begin{array}{cccccc}
0 & 0 & 1 & -1 & 0 & 0 \\
0 & 0 & 0 & 0 & 0 & 0 \\
0 & 1 & 0 & 0 & 0 & 0 \\
0 & 1 & 0 & 0 & 0 & 0 \\
1 & 0 & 0 & 0 & 0 & 0 \\
-1 & 0 & 0 & 0 & 0 & 0
\end{array}\right)\left(\begin{array}{l}
V_{x} \\
V_{w} \\
I_{y^{+}} \\
I_{y^{-}} \\
I_{z^{+}} \\
I_{z^{-}}
\end{array}\right)
$$

The circuit of proposed SRCO is drawn in Figure 2.

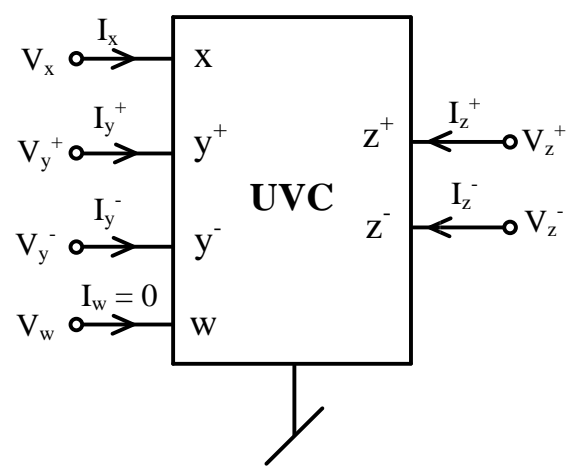

(a)

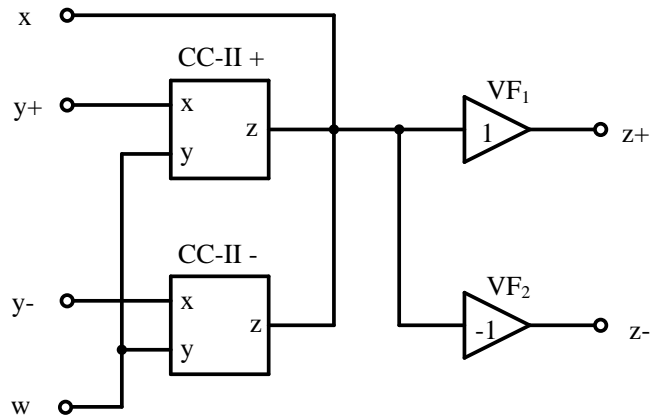

(b)

Figure 1. (a) Symbolic notation of UVC; (b) Equivalent circuit model of UVC [12]. 


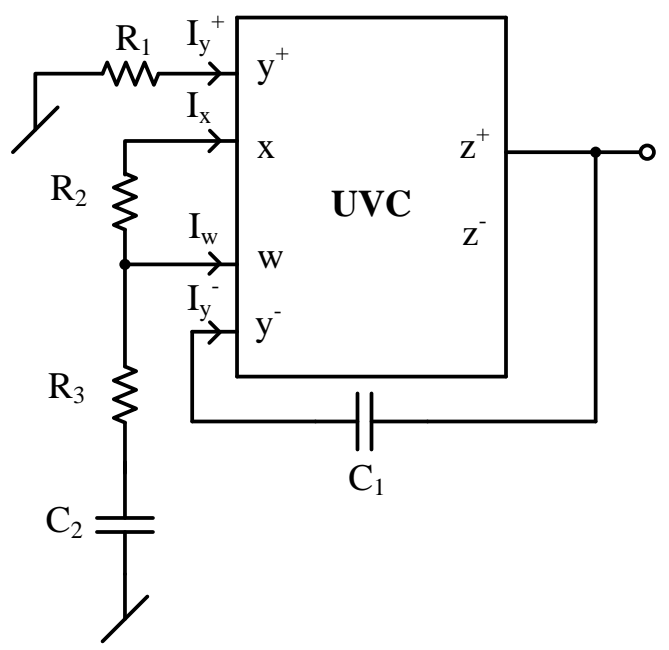

Figure 2. Proposed SRCO using single UVC.

Routine circuit analysis (assuming ideal UVC) of Figure 2 yields following expressions for characteristic Equation (CE) is given as:

CE: $s^{2} C_{1} C_{2}+s \frac{C_{2}}{R_{1} R_{2}}\left(R_{3}-R_{1}\right)+\frac{1}{R_{1} R_{2}}=0$

Thus, the condition of oscillation (CO) and frequency of oscillation (FO) are given by

$$
\begin{aligned}
& \text { CO: }\left(\frac{R_{3}}{R_{1}}-1\right) \leq 0 \\
& \text { FO: } \omega_{0}=\sqrt{\frac{1}{C_{1} C_{2} R_{1} R_{2}}}
\end{aligned}
$$

\section{Non-Ideal Analysis and Sensitivity Performance}

Taking into account the non-idealities of UVC, the relationship between the port voltages and currents is shown by the hybrid matrix:

$$
\left(\begin{array}{c}
I_{x} \\
I_{w} \\
V_{y^{+}} \\
V_{y^{-}} \\
V_{z^{+}} \\
V_{z^{-}}
\end{array}\right)=\left(\begin{array}{cccccc}
0 & 0 & \alpha_{1} & -\alpha_{2} & 0 & 0 \\
0 & 0 & 0 & 0 & 0 & 0 \\
0 & \delta_{1} & 0 & 0 & 0 & 0 \\
0 & \delta_{2} & 0 & 0 & 0 & 0 \\
\gamma_{1} & 0 & 0 & 0 & 0 & 0 \\
-\gamma_{2} & 0 & 0 & 0 & 0 & 0
\end{array}\right)\left(\begin{array}{c}
V_{x} \\
V_{w} \\
I_{y^{+}} \\
I_{y^{-}} \\
I_{z^{+}} \\
I_{z^{-}}
\end{array}\right)
$$

where $\alpha_{j}=1-\varepsilon_{i j}$ and $\delta_{j}=1-\varepsilon_{v 1 j}, \gamma_{j}=1-\varepsilon_{v 2 j}$ for $j=1,2$. Here $\varepsilon_{i j}\left(\left|\varepsilon_{i j}\right| \ll 1\right)$ and $\varepsilon_{v 1 j}, \quad \varepsilon_{v 2 j} \quad \varepsilon_{i j}\left(\left|\varepsilon_{v 1 j}\right|,\left|\varepsilon_{v 2 j}\right| \ll 1\right)$ represent the current and voltage tracking errors of the UVC respectively. The parasitic present on the low impedance ports $\left(y^{+}, y^{-}, z^{+}, z^{-}\right)$are quite low as compared to the resistances on the other ports ( $w$ and $x$ ) [11]. After considering the non-idealities of the UVC, given by the hybrid matrix of Equation (6), the characteristic Equation (CE), CO and $\mathrm{FO}$ are given as: 
The various sensitivities of FO can be found as

$$
\begin{gathered}
S_{C_{1}}^{\omega_{0}}=S_{C_{2}}^{\omega_{0}}=S_{R_{1}}^{\omega_{0}}=S_{\alpha_{2}}^{\omega_{0}}=-\frac{1}{2}, \\
S_{R_{2}}^{\omega_{0}}=-\frac{1}{2}\left(\frac{\gamma_{1} R_{2}}{\gamma_{1} R_{2}+\gamma_{1} R_{3}-\delta_{2} R_{3}}\right), \\
S_{\gamma_{1}}^{\omega_{0}}=-\frac{1}{2} \frac{S_{\alpha_{1}}^{\omega_{0}}=\frac{1}{2}}{\left(\gamma_{1} R_{2}+\gamma_{1} R_{3}-\delta_{2} R_{3}\right)}, \\
S_{\delta_{2}}^{\omega_{0}}=\frac{1}{2}\left(1+\frac{R_{3}}{\alpha_{1} \delta_{2}\left(\gamma_{1} R_{2}+\gamma_{1} R_{3}-\delta_{2} R_{3}\right)}\right) \\
S_{R_{3}}^{\omega_{0}}=-\frac{1}{2}\left(\frac{R_{3}\left(\gamma_{1}-\delta_{2}\right)}{\alpha_{1} \delta_{2}\left(\gamma_{1} R_{2}+\gamma_{1} R_{3}-\delta_{2} R_{3}\right)}\right)
\end{gathered}
$$

In the ideal case, the various sensitivities of $\omega_{0}$ with respect to $R_{1}, R_{2}, C_{1}$ and $C_{2}$ are given as

$$
S_{C_{1}}^{\omega_{0}}=S_{C_{2}}^{\omega_{0}}=S_{R_{1}}^{\omega_{0}}=S_{R_{2}}^{\omega_{0}}=-\frac{1}{2}
$$

\section{Simulation}

To confirm the validity of the presented SRCO, the circuit was simulated using SPICE. The voltage and current values selected for CMOS implementation of UVC are $\pm 1.9 \mathrm{~V}$ and $100 \mu \mathrm{A}$, respectively. The passive elements were chosen as $C_{1}=C_{2}=\operatorname{lnF}, R_{1}=R_{2}=2.4 \mathrm{k} \Omega$ and $R_{3}=2.331 \mathrm{k} \Omega$. The transient and steady state response of the proposed SRCO for the selected passive components are shown in Figure 3 and Figure 4 respectively. The SPICE simulated frequency response of the SRCO is shown in Figure 5. CMOS implementation of universal voltage conveyor is shown in Figure 6 and the W/L ratios of transistors used in Figure 6 are given in Table 1 [15]. CMOS UVC was implemented $0.18 \mu \mathrm{m}$ TSMC CMOS model parameters [17].

\section{Conclusion}

A new voltage-mode single-resistance controlled sinusoidal oscillator employing single UVC has been proposed. The presented SRCO circuit employs single UVC, two capacitors and three resistors. The presented configuration offers (1) independent control of frequency of oscillation can be controlled by resistance 


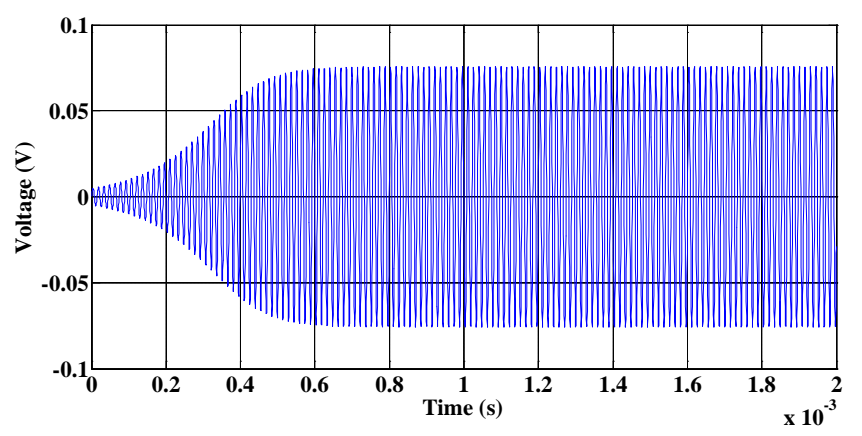

Figure 3. Transient response of the proposed SRCO.

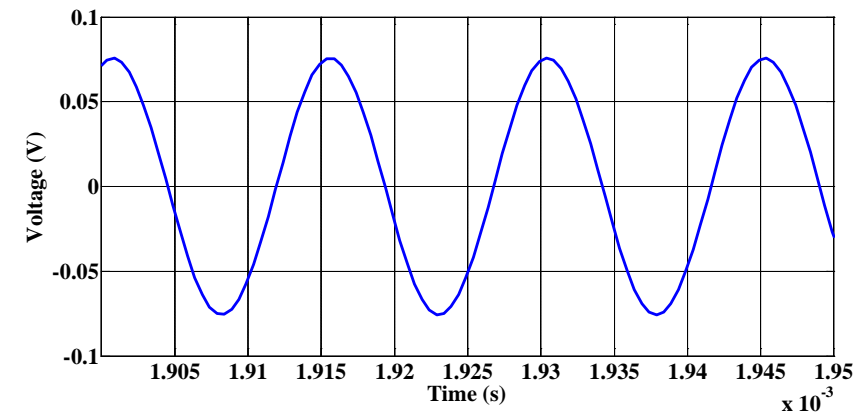

Figure 4. Steady state response of the proposed SRCO.

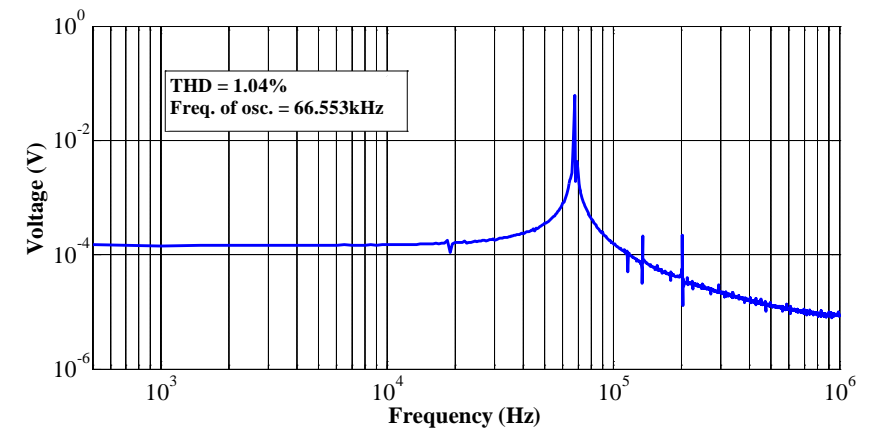

Figure 5. Frequency spectrum of the proposed SRCO.

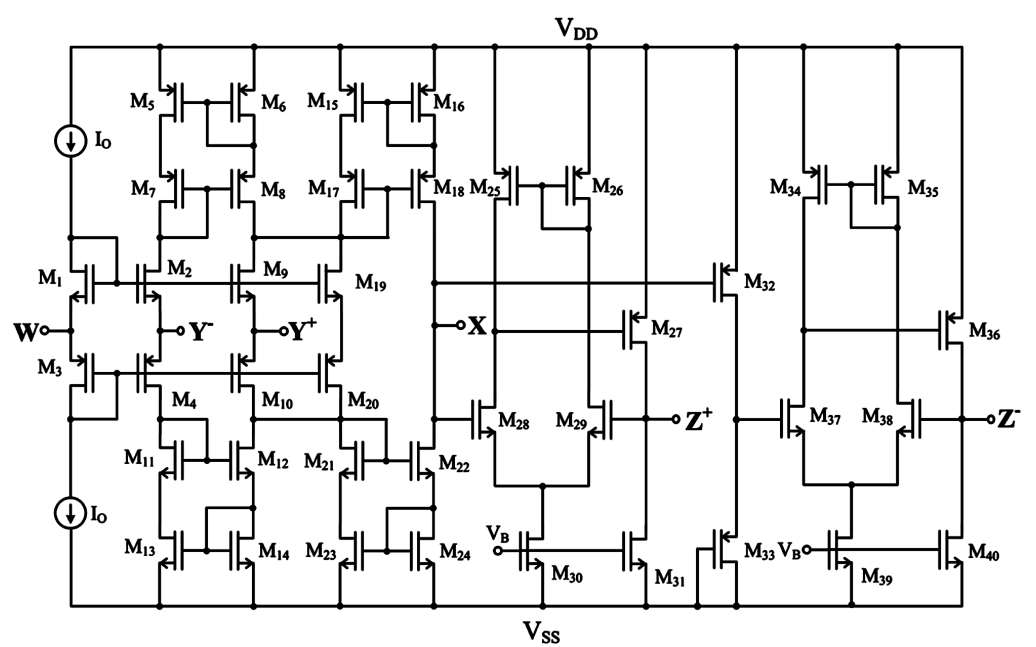

Figure 6. Implementation of CMOS structure of UVC, $V_{\mathrm{DD}}=-V_{\mathrm{SS}}=1.9 \mathrm{~V}, I_{\mathrm{o}}=100 \mu \mathrm{A}$. 
Table 1. The aspect ratios $(W / L)$ of transistors used in Figure 6.

\begin{tabular}{ccc}
\hline Transistors & $W(\mu \mathrm{m})$ & $L(\mu \mathrm{m})$ \\
\hline M5-M8, M10, M15-M18, M20 & 14.0 & 0.7 \\
M3, M4 & 28 & 0.7 \\
M25, M26, M34, M35 & 4.0 & 0.5 \\
M27, M36 & 10.0 & 0.5 \\
M32, M33 & 2.1 & 1.0 \\
M1, M2 & 14.0 & 0.7 \\
M9, M11-M14, M19, M21-M24 & 28 & 0.7 \\
M28, M29, M37, M38 & 0.8 & 05 \\
M30, M31, M39, M40 & 10 & 0.5 \\
\hline
\end{tabular}

$\mathrm{R}_{2}$ and condition of oscillation can be adjusted by resistance $R_{3}$, (2) low passive sensitivities. Simulation results using $0.18 \mu \mathrm{m}$ TSMC CMOS technology have been presented to confirm the workability of the proposed new SRCO. This paper thus added a new application configuration to the existing repertoire of UVC based application circuits. For future one can reduce the passive elements or transistors in the device (UVC).

\section{References}

[1] Biolek, D., Senani, R., Biolkova, V. and Kolka, Z. (2008) Active Elements for Analog Signal Processing: Classification, Review, and New Proposals. Radioengineering, 17, 15-32.

[2] Pushkar, K.L., Singh, G. and Goel, R.K. (2017) CMOS VDIBAs-Based Single-Resistance-Controlled Voltage-Mode Sinusoidal Oscillator. Circuits and Systems, 8, 14-22. https://doi.org/10.4236/cs.2017.81002

[3] Senani, R. and Bhaskar, D.R. (1991) Single Op-Amp Sinusoidal Oscillators Suitable for Generation of Very Low Frequencies. IEEE Transactions on Instrumentation and Measurement, 40, 777-779. https://doi.org/10.1109/19.85353

[4] Bhaskar, D.R. and Senani, R. (2006) New CFOA-Based Single-Element-Controlled Sinusoidal Oscillators. IEEE Transactions on Instrumentation and Measurement, 55, 2014-2021. https://doi.org/10.1109/TIM.2006.884139

[5] Senani, R. (1985) New Types of Sine Wave Oscillators. IEEE Transactions on Instrumentation and Measurement, 34, 461-463. https://doi.org/10.1109/TIM.1985.4315370

[6] Pushkar, K.L., Bhaskar, D.R. and Prasad, D. (2013) Single-Resistance Controlled Sinusoidal Oscillator Using Single VD-DIBA. Active and Passive Electronic Components, 2013, Article ID 971936. https://doi.org/10.1155/2013/971936

[7] Pushkar, K.L., Goel, R.K., Gupta, K., Vivek, P. and Ashraf, J. (2016) New VD-DIBABased Single-Resistance-Controlled Sinusoidal Oscillator. Circuits and Systems, 7, 4145-4153. https://doi.org/10.4236/cs.2016.713341

[8] Herencsar, N., Koton, J. and Vrba, K. (2011) KHN-Equivalent Voltage-Mode Filters Using Universal Voltage Conveyors. AEU-International Journal of Electronics and Communications, 65, 154-160. https://doi.org/10.1016/j.aeue.2010.02.005

[9] Minarcik, M. and Vrba, K. (2007) Continuous-Time Multi-Functional Filters with 
Wide Bandwidth Using Universal Voltage Conveyors. Proceedings of the 6 th International Conference on Networking.

[10] Pushkar, K.L. and Gupta, K. (2017) MISO-Type Voltage-Mode Universal Biquadratic Filter Using Single Universal Voltage Conveyor. Circuits and Systems, 8, 227236. https://doi.org/10.4236/cs.2017.89015

[11] Herencsar, N., Koton, J. and Vrba, K. (2009) A New Electronically Tunable VoltageMode Active-C Phase Shifter Using UVC and OTA. IEICE Electron. Express, 6, 1212-1218.

[12] Minarcik, M. and Vrba, K. (2006) Low-Output and High-Input Impedance Frequency Filters Using Universal Voltage Conveyor for High-Speed Data Communication Systems. Proceedings of International Conference on Networking, April 2329 2006, Mauritius.

[13] Pushkar, K.L., Gupta, K. and Vivek, P. (2017) A New Voltage-Mode Universal Biquadratic Filter Using Single UVC. Circuits and Systems, 8.

[14] Sponar, R. and Vrba, K. (2006) Measurements and Behavioral Modeling of Modern Conveyors. International Journal of Computer Science and Network Security, 6, 5765.

[15] Herencsar, N., Koton, J., Vrba, K. and Minaei, S. (2011) Electronically Tunable MOSFET-C Voltage-Mode All Pass Filter Based on Universal Voltage Conveyor. Communication Software and Networks (ICCSN), May 27-29 2011, Xi'an, 442-445. https://doi.org/10.1109/ICCSN.2011.6014931

[16] Koton, J., Herencsa, N. and Vrba, K. (2012) Current- and Voltage-Mode ThirdOrder Quadrature Oscillator. 2012 13th International Conference on Optimization of Electrical and Electronic Equipment (OPTIM), May 24-26 2012, Brasov, 12031206. https://doi.org/10.1109/OPTIM.2012.6231795

[17] Minaei, S. and Yuce, E. (2010) Novel Voltage-Mode All-Pass Filter Based on Using DVCCs. Circuits System and Signal Processing, 29, 391-402.

https://doi.org/10.1007/s00034-010-9150-3 